\title{
The Energy and Zenith Angle Distribution of Upward Going Muons and Neutrino Oscillations
}

\author{
Paolo Lipari and Maurizio Lusignoli \\ Dipartimento di Fisica, Università di Roma "la Sapienza", \\ and I.N.F.N., Sezione di Roma, Piazzale A. Moro 2, \\ I-00185 Roma, Italy \\ (Received 5 December 1997)
}

\begin{abstract}
The energy and zenith angle distribution of neutrino induced, upward going muons can give direct information on the presence of $\nu$-oscillations in precisely the range of parameters suggested as a solution of the atmospheric neutrino problem. We discuss here the uncertainties in the theoretical prediction. The shape of the zenith angle distribution of the muon flux is quite insensitive to modifications of the theoretical input and is a good probe for the existence of neutrino oscillations. We conclude that the existing data sample on $\nu$-induced muons has the statistical power to confirm or refute the $\nu$-oscillation solution of the atmospheric neutrino problem.
\end{abstract}

PACS numbers: 14.60.Pq, 96.40.Tv

The recent results of the Superkamiokande (SK) experiment [1] on atmospheric neutrinos confirm the existence of a problem [2] in the relative numbers of $\nu_{e}$ and $\nu_{\mu}$ induced events, that may be interpreted as an indication of neutrino oscillations with large mixing. The favoured $\Delta m^{2}$ has however been shifted to values lower than those suggested by the older Kamiokande results [3]. The results of the Chooz experiment [4] exclude large amplitude oscillations of electron antineutrinos into other antineutrinos in the region of interest for $\Delta m^{2}$, however $\nu_{\mu} \leftrightarrow \nu_{\tau}$ (or $\nu_{\mu} \leftrightarrow \nu_{s}$ where $\nu_{s}$ is a sterile neutrino) oscillations remain a viable solution of the problem. A dedicated long-baseline experiment could provide a clear confirmation of the existence of oscillations [5]; the actual detection of $\tau$-leptons produced in the detector would be especially striking, however this would only be possible with a high energy neutrino beam and the distance needed to observe such an effect would become prohibitively long if $\Delta m^{2} \lesssim 10^{-3} \mathrm{eV}^{2}$. It is therefore very desirable to obtain clearer indications on the value of the parameter $\Delta m^{2}$.

In this work, we rediscuss the information that can be obtained from the analysis of $\nu$-induced upward going muons. In particular we consider in detail the shape of the zenith angle distribution and suggest that it is a sensitive and model independent probe of the existence of neutrino oscillations. In the presence of $\nu$-oscillations the muon flux is reduced in its absolute value, its energy spectrum is distorted, and the zenith angle distribution is deformed. Since the $\nu$ induced flux can be measured only in half of the solid angle, and the deviations from the up-down symmetry $\phi_{\mu}(E, \theta)=\phi_{\mu}(E, \pi-\theta)$, valid in the absence of oscillations, cannot be measured, the interpretation of the data requires a comparison with a calculated prediction, and the estimate of theoretical uncertainties in the calculation is crucial.

The absolute value of the predicted flux is affected by a large uncertainty $\sim 20 \%$ [6]. This uncertainty is not relevant in the study of the shape of the muon energy spectrum. Since no underground muon detector has used a magnetic field, a measurement of the energy can only be obtained for the muons that stop in the detector using the particle range. In the presence of oscillations the flux of low energy muons is suppressed more, and the ratio $r=N_{s} / N_{p}$ of the numbers of 'stopping' and 'passing' muon events is reduced with respect to the no-oscillation prediction. From a measurement of $r$, the IMB collaboration [7] has obtained an exclusion region in the $\left(\Delta m^{2}, \sin ^{2} 2 \theta_{\mu \tau}\right)$ plane that is in sharp contradiction with the allowed region obtained by SK [1] from the analysis of the events with $\nu$ interactions inside the detector. The significance of the discrepancy is much reduced in the critical reanalysis of [8], where the prediction for the value of $r$ in the absence of oscillations is increased by $\sim 12 \%$ with respect to the calculation used in the original IMB work. The SK detector, with a larger effective area, larger containement volume and better spatial and energy resolution can provide a new and more accurate measurement of the stop/pass ratio.

The direction of the muons is well measured in underground detectors and the shape of the angular distribution can be studied in detail and compared to different predictions (with or without oscillations) 
using statistical tests. For illustration and the sake of clarity in this work we will simply use the ratio of the of 'horizontal' and 'vertical' muon events, a single number, to describe the shape of the distribution:

$$
S=\int_{-\frac{1}{2}}^{0} d \cos \theta \frac{d N}{d \cos \theta} \cdot\left[\int_{-1}^{-\frac{1}{2}} d \cos \theta \frac{d N}{d \cos \theta}\right]^{-1} .
$$

In the presence of oscillations the flux of vertical muons is supressed more (because the quasi-collinear parent neutrinos have longer pathlengths) and $S$ increases, by as much as $30 \%$ for $\Delta m^{2}$ in the region of interest and maximal mixing. The separate measurement of the quantities $S_{s}$ and $S_{p}$, related to the angular distributions for 'stopping' and 'passing' muons, results in an improvement of the sensitivity, a better control of systematic effects, and in case of a positive signal a more precise indication of the value of $\Delta m^{2}$. We estimate the uncertainty in the predicted value of $S, S_{s}$ and $S_{p}$ as $\lesssim 4 \%$.

In previous theoretical discussions of stopping muons [9]6] it was assumed for simplicity that stopping muons are in the energy interval $E_{\min } \leq E_{\mu} \leq E^{*}$, and passing muons have energies $E_{\mu} \geq E^{*}$. This approximation is clearly not realistic. For simple geometrical reasons, the energy interval where stopping muons can be identified depends on the zenith angle; moreover in general, even for a fixed zenith angle, muons of the same energy can either stop in or pass through the detector, depending on their trajectory. In general the rates of stopping and passing muons in the detector can be estimated as:

$$
\frac{d N_{s(p)}}{d \cos \theta}=\int_{0}^{\infty} d E_{\mu} \phi_{\mu}\left(E_{\mu}, \cos \theta\right) A_{s(p)}\left(E_{\mu}, \theta\right)
$$

where $\phi_{\mu}\left(E_{\mu}, \cos \theta\right)$ is the differential flux of upward going muons of energy $E_{\mu}$ and zenith angle $\theta$, and $A_{s(p)}\left(E_{\mu}, \theta\right)$ is the detector acceptance for stopping (passing) muons. A detailed calculation of the acceptances as a function of muon energy and direction is only possible for the experimental collaboration. In the following, considering the case of the SK detector, as a first approximation we describe it as a homogeneous cylindrical volume of radius $R=16.9 \mathrm{~m}$ and height $H=36.2 \mathrm{~m}[10$ and approximate the muon trajectories as straight lines with length equal to the muon range in water; in this way the calculation of the acceptance is reduced to an elementary geometry problem.

The requirement that the muon track be entirely contained in the detector volume gives:

$$
A_{s}\left(E_{\mu}, \theta\right)=A\left(L\left(E_{\mu}\right), \theta\right)
$$

where $L(E)$ is the muon range in water and

$$
A(L, \theta)=2 R H \sin \theta \sqrt{1-x^{2}}+2 R^{2}|\cos \theta|\left[\cos ^{-1} x-3 x \sqrt{1-x^{2}}\right] \Theta\left[L_{\max }(\theta)-L\right],
$$

with $x=L \sin \theta / 2 R$ and $L_{\max }(\theta)=\min [2 R / \sin \theta, H /|\cos \theta|]$. The acceptance for stopping muons is non-zero only when the muon range is in the interval $L_{\min } \leq L(E) \leq L^{*}$, where $L_{\min }$ is the minimum track length required for detection ( $7 \mathrm{~m}$ in the first analysis of SK [10]), and $L^{*}$ is the maximum linear dimension of the detector ( $49.5 \mathrm{~m}$ in SK); this corresponds to the energy interval $1.45 \leq E_{\mu} \leq 10.63 \mathrm{GeV}$. The acceptance for passing muons is given by:

$$
A_{p}\left(E_{\mu}, \theta\right)=A\left(L_{\min }, \theta\right)-A_{s}\left(E_{\mu}, \theta\right) .
$$

In Table I we give our estimates of some quantities of interest (total event rate, $r, S_{s}, S_{p}$ ) calculated in the absence of oscillations with different choices of the theoretical input, as described in the first three columns. Column 1 indicates the choice of a neutrino flux from those available in the literature [11 17]. In column 2 the label LLS indicates that the contributions of quasi-elastic scattering and of single pion production to the neutrino cross sections have been explicitely included [8], while the label DIS indicates that the neutrino cross section is described by the formulae of deep inelastic scattering; the more accurate treatment of the lowest multiplicity channels in the LLS option results in a higher total flux and a higher stop/pass ratio $r$. The third column indicates the choice made for the Parton Distribution Functions (PDF), using the label defined in the library code PDFLIB [22]. The first row in table I contains the absolute values of the predictions for our reference model (Bartol $\phi_{\nu}$ [11], PDF by [23] and LLS [8] cross sections); in the other rows we report ratios with respect to the reference values. 
Our reference model predicts a rate of $521 \mathrm{yr}^{-1}$ of passing muons with track length larger than $7 \mathrm{~m}$. This can be compared with a preliminary result obtained in 229 days of live time of $425 \pm 26 \mathrm{yr}^{-1} 10$. We also predict a rate of $218 \mathrm{yr}^{-1}$ stopping muons. It is important to observe that this prediction is quite sensitive to the value of the cut $L_{\min }$, because the muon flux is steeply falling with energy in this region; a lower (higher) value $L_{\min }=5(9) \mathrm{m}$ results in a stopping rate of $269(169) \mathrm{yr}^{-1}$. It will be therefore important to consider in detail the resolution in the measurement of the stopping point of the muon tracks. The distribution of the length $L$ of the stopping tracks is a measurement of the muon spectrum in the low energy region; however this region is narrow, and with the expected event rates the possible distortions of the $d N / d L$ distributions will be hard to detect.

Our predictions for the parameters $S_{s}$ and $S_{p}$ that describe the shape of the zenith angle distributions of stopping and passing muons are 1.45 and 1.63. In both cases there is an excess of horizontal muons, and the anisotropy is stronger for the higher energy (passing) muons, $S_{p}>S_{s}$. The anisotropy of the muon flux and its energy dependence originate from the zenith angle dependence of the neutrino flux. The angular distribution is approximately isotropic for low energy neutrinos, and it develops a stronger and stronger dependence on the zenith angle with increasing neutrino energy. This can be understood by considering the larger decay probability for high energy mesons and muons traveling in a direction near to the horizontal.

The spread of numbers in the columns of table I can be used as an estimate of the uncertainty in the prediction for the different observables. We estimate $\Delta r \sim 8 \%, \Delta S_{s} \sim 3 \%$ and $\Delta S_{p} \sim 4 \%$. The MMK [17] neutrino flux gives $S_{s(p)}$ larger than our reference value by a factor 1.16 (1.13), in contrast to all other models, and we disregard this prediction in the following.

To explore the possible effects of modifications in the theoretical inputs of the neutrino flux calculation, we have repeated the $\nu$-flux calculation of 15] including some extreme changes in the initial assumptions. In [15] the primary cosmic ray flux has a power law energy spectrum $E^{-\alpha}$ with $\alpha=2.7$; steepening (flattening) the spectrum using $\alpha=2.8$ (2.6) increases (decreases) the stop/pass ratio $r$ by $+26 \%$ ($21 \%$ ), correspondingly the $\cos \theta$ distribution becomes flatter (steeper) with the much smaller variation of $\mp 1 \%$ in $S_{s}$ and $\mp 3 \%$ in $S_{p}$. Increasing (reducing) the $\mathrm{K}$ meson yield by a factor 1.5 changes $r$ by $\pm 8 \%, S_{s}$ by $\pm 3 \%$ and $S_{p}$ by $\pm 4 \%$. These tests indicate that the prediction on the shape of the angular distribution is quite stable.

The effects of neutrino oscillations on the observables that we are discussing are illustrated in the figures. In fig. 1 the solid curve shows the dependence on $\Delta m^{2}$ for maximal mixing of the ratio $r$ between the number of stopping and passing events, for our reference model. The error bars indicate only statistical errors estimated for two years of SK data-taking (i.e. in a few months from now). In the region $10^{-3} \leq \Delta m^{2}\left(\mathrm{eV}^{2}\right) \leq 10^{-2}$, favoured by the anomaly in the contained and semi-contained events, the stop/pass ratio has a value $r \lesssim 0.30$ to be compared with a no-oscillation prediction 0.42 . It is apparent that in this region of $\Delta m^{2}$, for large mixing, the statistical significance of the effects of oscillations is clear (as large as $\sim 7 \sigma$ ), and the theoretical uncertainty of the prediction is likely to be the most important source of error. With the estimate of $8 \%$ for the theoretical error and reasonable systematic errors in the experiment, an effect should be clearly visible if neutrino oscillations are indeed the reason of the atmospheric neutrino anomaly. However, the suppression of $r$ is approximately the same for $\Delta m^{2}=10^{-3}$ or $10^{-2} \mathrm{eV}^{2}$ and therefore with an analysis of this quantity it will not be possible to resolve the present uncertainty on $\Delta m^{2}$. In fig. 1 we also included (dashed line) the $\Delta m^{2}$ dependence for maximal mixing of $r$ for $\nu_{\mu}$ oscillations into sterile neutrinos, the curve being shifted because of the matter effects 9 .

We have plotted in fig. 2 versus $\Delta m^{2}$, again for maximal mixing, the quantities $S_{s}$ and $S_{p}$ that are a measure of the shape of the angular distributions. The error bars indicate the estimated statistical errors, which in this case are likely to be the dominant source of error. In the presence of neutrino oscillations both $S_{s}$ and $S_{p}$ are larger than the no-oscillation expectation because of the larger suppression of the vertical flux. For a fixed value of the mixing parameter, the effect of oscillations on $S_{s}\left(S_{p}\right)$ has a broad maximum for $\Delta m^{2} \simeq 0.7 \cdot 10^{-3}\left(10^{-2}\right) \mathrm{eV}^{2}$. Qualitatively we can expect that the angular distortion produced by $\nu$-oscillations is maximum when

$$
\Delta m^{2} \sim \frac{2 \pi}{R_{\oplus}}\left\langle E_{\nu}\right\rangle,
$$


where $R_{\oplus}$ is the earth radius and $\left\langle E_{\nu}\right\rangle$ is the typical energy of the neutrinos that produce the upward going muon signal; for smaller values of $\Delta m^{2}$ the oscillations do not have time to develop, for larger values the effects of oscillations suppress the flux equally for all directions. The signals of passing and stopping muons are produced by neutrinos with median energy $\left\langle E_{\nu}^{s(p)}\right\rangle \simeq 8(100) \mathrm{GeV}$ and this is reflected in the positions of the maxima in fig. 2. We also note that for large mixing and $\Delta m^{2} \sim 0.2-1 \cdot 10^{-3} \mathrm{eV}^{2}$ one should have $S_{s}>S_{p}$, an essentially model-independent signal of oscillations, albeit with low statistical significance. The matter effects, present in the $\nu_{\mu} \leftrightarrow \nu_{s}$ case, induce a detectable but different deformation in the angular distribution; the parameters $S_{s, p}$ are not a good measure of the effect in this case, since the suppression for 'horizontal' and 'vertical' events are approximately equal, after integration over the solid angle.

In fig. 3 we present in the usual $\left(\Delta m^{2}, \sin ^{2} 2 \theta_{\mu \tau}\right)$ plane the regions that may be excluded at $95 \%$ c.l. after two years of SK running assuming that the experiment measures the central value of the expectations (without neutrino oscillations), by a measurement of $r$ (solid line), $S_{s}$ (dashed) and $S_{p}$ (dot-dashed). In order to produce this plot, we (arbitrarily) combined quadratically the expected statistical error and the theoretical uncertainty. Conversely, the effect of atmospheric $\nu_{\mu} \leftrightarrow \nu_{\tau}$ oscillations for representative parameter values, maximal mixing and $\Delta m^{2}=10^{-3}\left(10^{-2}\right) \mathrm{eV}^{2}$, would result in a reduction in the stop/pass ratio $r$ of $30(26) \%$, with a deviation of $3.2(2.7) \sigma$ 's including the theoretical error, and shape parameters $S_{s}=2.14 \pm 0.27(1.55 \pm 0.21)$ and $S_{p}=1.82 \pm 0.12(2.13 \pm 0.17)$, that correspond to 2.5 and 1.6 (0.5 and 2.9) standard deviation effects. In both cases the signal should be detectable, and the analysis offers a rough measurement of the $\Delta m^{2}$ parameter.

Experimental results on upward going muons have already been presented. Baksan [18], MACRO [19], Kamiokande [20], IMB [21, ], and Superkamiokande [10], have collected large statistical samples of 'passing' events. The results for the total rates are inconclusive, with respect to the oscillations, because of the large errors and theoretical uncertainties. All these experiments have presented measurements of the muon flux above a threshold energy as a function of the zenith angle. We point out that the extraction of a detector independent flux from the measured passing muon rates is both non trivial and model dependent, because no detector has a well defined and angle independent threshold, and one needs corrections that make use of the theoretical prediction. Since this is normally done assuming the absence of oscillations, the fluxes can be used to test this hypothesis, but great care has to be taken in the extraction of oscillation parameters. The comparison of event rates with detailed MonteCarlo predictions is free from these interpretation problems, that is why we preferred to make a rough estimate of the acceptance and discuss predictions of directly measurable quantities. For similar reasons, it seems dangerous to reduce the data of different experiments to a common threshold, as was done in a recent analysis [24]. The experimental results are summarized in table II, where we give the ratio $S_{\phi}^{\exp }$ of the vertical and horizontal fluxes obtained from the published data, and our no-oscillation prediction $S_{\phi}^{t h}$. All experiments measure a value larger than the prediction. This could be interpreted as an effect of neutrino oscillations, but such a conclusion would be premature. The fit of the shape of the angular distributions for the individual experiments is poor even after the inclusion of oscillations; moreover a comparison between experiments shows that the normalizations and detailed shapes of the data are not in good agreement with each other, suggesting the presence of unforeseen systematic effects (see [24] for a discussion). Nonetheless the qualitative result of an excess of horizontal muons in all experiments is a hint that should be studied in more detail.

In summary, a better measurement of upward going muons, as it will be possible in the SuperKamiokande detector, can test the neutrino oscillation solution of the (semi)contained atmospheric neutrino problem. Sensitive quantities are: the ratio of stopping to passing muons, already noted and discussed, and the shapes of angular distributions, that are affected by an even smaller theoretical uncertainty.

[1] Y. Totsuka, to be published in the proceedings of the Lepton Photon Conference, Hamburg, july 1997, 
available at http://www-sk.icrr.u-tokyo.ac.jp/doc/sk/pub/lp97.ps

[2] See for example T. K. Gaisser, in Neutrino '96, Proceedings of the 17th Conference on Neutrino Physics and Astrophysics, Helsinki, 1996.

[3] Kamiokande collaboration: K.S. Hirata et al. Phys. Lett. B 205, 416 (1988), ibid. 280, 146 (1992); Y. Fukuda et al. Phys. Lett. B 335, 237 (1994).

[4] Chooz collaboration: M. Apollonio et al., hep-ex/971102.

[5] For documentation on the numerous proposals, see: www.hep.anl.gov/NDK/Hypertext/long_baseline.html

[6] W. Frati, T. K. Gaisser, A. K. Mann, and T. Stanev, Phys. Rev. D 48, 1140 (1993).

[7] IMB Collaboration: R. Becker-Szendy et al., Phys. Rev. Lett. 69, 1010 (1992).

[8] P. Lipari, M. Lusignoli, and F. Sartogo, Phys.Rev.Lett. 74, 4384 (1995).

[9] E. Akhmedov, P. Lipari, and M. Lusignoli, Phys. Lett. B 300, 128 (1993).

[10] SuperKamiokande Collaboration, presented by J. G. Learned in ICRC '97, Durban, South Africa, astro$\mathrm{ph} / 9705197$.

[11] [Bartol] V. Agrawal, T. K. Gaisser, P. Lipari, and T. Stanev, Phys. Rev. D 53 1314, (1996).

[12] [HKKM] M. Honda, T. Kajita, K. Kasahara, and S. Midorikawa, Phys. Rev. D 52, 4985 (1995);

[13] [BDZ] A. V. Butkevich, L. G. Dedenko, and I. M. Zheleznykh, Sov. J. Nucl. Phys. 50, 90 (1989).

[14] [Volkova] L.V. Volkova, Sov.J.Nucl.Phys. 31, 784 (1980).

[15] [Lipari] P. Lipari, Astropart. Phys. 1, 195 (1993).

[16] [FLUKA] G. Battistoni et al., to appear in the Proceedings of TAUP 97, LNGS, and private communication.

[17] [MMK] K. Mitsui, Y. Minorikawa, and H. Komori, Nuovo Cimento C 9, 995 (1986).

[18] Baksan Collaboration: M. M. Boliev et al., ICRC '95, Rome, Vol 1, p. 686; ibidem, p. 722.

[19] MACRO Collaboration: S. Ahlen et al., Phys. Lett. B 357, 481 (1995); F. Ronga et al., in Neutrino '96, Proceedings of the 17th Conference on Neutrino Physics and Astrophysics, Helsinki, 1996.

[20] Kamiokande Collaboration: A. Suzuki et al. in Proceedings of 7th International Workshop on Neutrino Telescopes, Venice, 1996, edited by M. Baldo Ceolin p. 263.

[21] IMB collaboration: D. W. Casper, in Proceedings of 3rd International Workshop on Neutrino Telescopes, Venice, 1991, edited by M. Baldo Ceolin p. 213.

[22] H. Plotow-Besch, "PDFLIB: The Parton Density Function Library," User's Manual, Version 7.07, available at the URL http://consult.cern.ch/writeup/pdflib.

[23] M. Gluck, E. Reya, and A. Vogt, Z.Phys. C 67, 433 (1995).

[24] G.L. Fogli, E. Lisi and A. Marrone, preprint BARI-TH-280-97, hep-ph/9708213. 
TABLE I. Predictions for observables related to upward going muon flux in the absence of oscillations for the SuperKamiokande detector.

\begin{tabular}{|c|c|c|c|c|c|c|}
\hline$\phi_{\nu}$ & $\sigma_{\nu}$ & PDF & Rate $\left(\mathrm{yr}^{-1}\right)$ & $r$ & $S_{s}$ & $S_{p}$ \\
\hline Bartol & LLS & GRV94LO & 738 & 0.42 & 1.45 & 1.63 \\
\hline$\overline{\mathrm{HKKM}}$ & $\overline{\mathrm{LLS}}$ & GRV94LO & 0.94 & 0.98 & $\overline{1.00}$ & $\overline{1.00}$ \\
\hline BDZ & LLS & GRV94LO & 1.04 & 1.08 & 1.00 & 1.02 \\
\hline Volkova & LLS & GRV94LO & 0.94 & 1.02 & 1.02 & 1.04 \\
\hline Lipari & LLS & GRV94LO & 0.95 & 0.98 & 1.01 & 1.00 \\
\hline FLUKA & LLS & GRV94LO & 1.00 & 0.93 & 1.01 & 1.02 \\
\hline MMK & LLS & GRV94LO & 1.00 & 1.09 & 1.16 & 1.13 \\
\hline$\overline{\text { Bartol }}$ & LLS & GRVLO & 0.94 & 1.00 & 1.00 & $\overline{1.00}$ \\
\hline Bartol & LLS & GRV94DIS & 0.98 & 0.99 & 1.00 & 1.00 \\
\hline Bartol & LLS & CTEQ4-23 & 1.05 & 0.98 & 1.00 & 1.00 \\
\hline Bartol & LLS & EHLQ2 & 0.90 & 1.02 & 1.00 & 1.00 \\
\hline Bartol & DIS & GRV94LO & 0.99 & 0.96 & 1.00 & $\overline{1.00}$ \\
\hline Bartol & DIS & GRVLO & 0.92 & 0.95 & 1.00 & 1.00 \\
\hline Bartol & DIS & EHLQ2 & 0.85 & 0.89 & 1.00 & 1.00 \\
\hline
\end{tabular}

TABLE II. Measurements of the shape of the zenith angle distribution of the $\nu$-induced muon flux. The theoretical expectation is based on our reference model.

\begin{tabular}{lccc}
\hline \hline Detector & $E_{t}(\mathrm{GeV})$ & $S_{\phi}^{\text {exp }}$ & $S_{\phi}^{\text {th }}$ \\
\hline Baksan & 1.0 & $1.82 \pm 0.18$ & 1.50 \\
MACRO $^{\mathrm{a}}$ & 1.0 & $2.97 \pm 0.65$ & 1.50 \\
IMB & 1.8 & $1.79 \pm 0.18$ & 1.53 \\
Kamiokande & 3.0 & $1.91 \pm 0.21$ & 1.56 \\
SK & 6.0 & $1.83 \pm 0.23$ & 1.60 \\
\hline \hline
\end{tabular}

$\mathrm{a}_{\text {The Macro detector has a very low acceptance for near to horizontal }}$ muons and the datum in the first angular bin has a very large error, that propagates into the ratio of fluxes. If one neglects the first bin and extrapolates from the others a ratio $S_{\phi}$ similar to the other rows is obtained. 


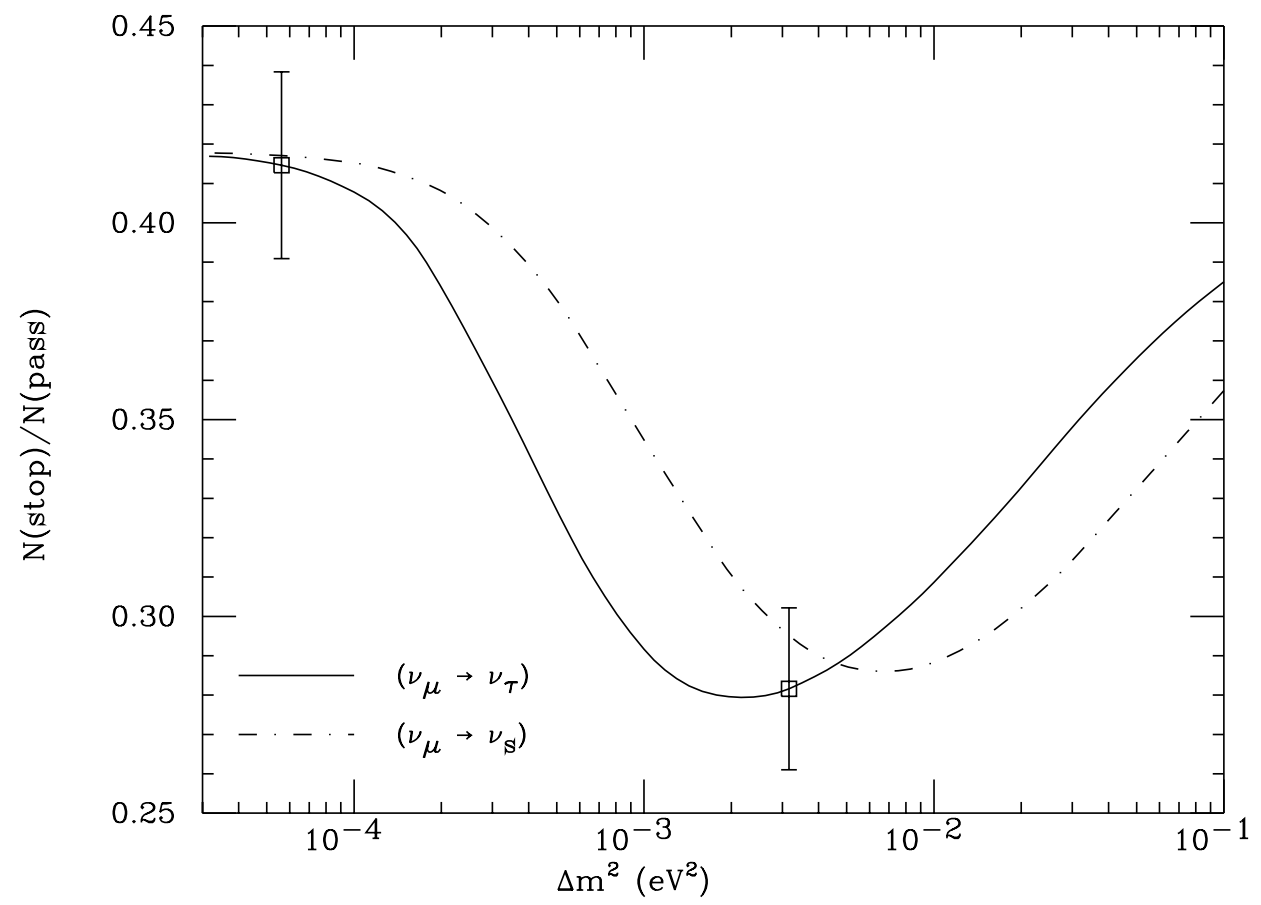

FIG. 1. Ratio of the rates of 'stopping' and 'passing' muons (with $L_{\min }=7 \mathrm{~m}$ ) in SuperKamiokande for maximal mixing.

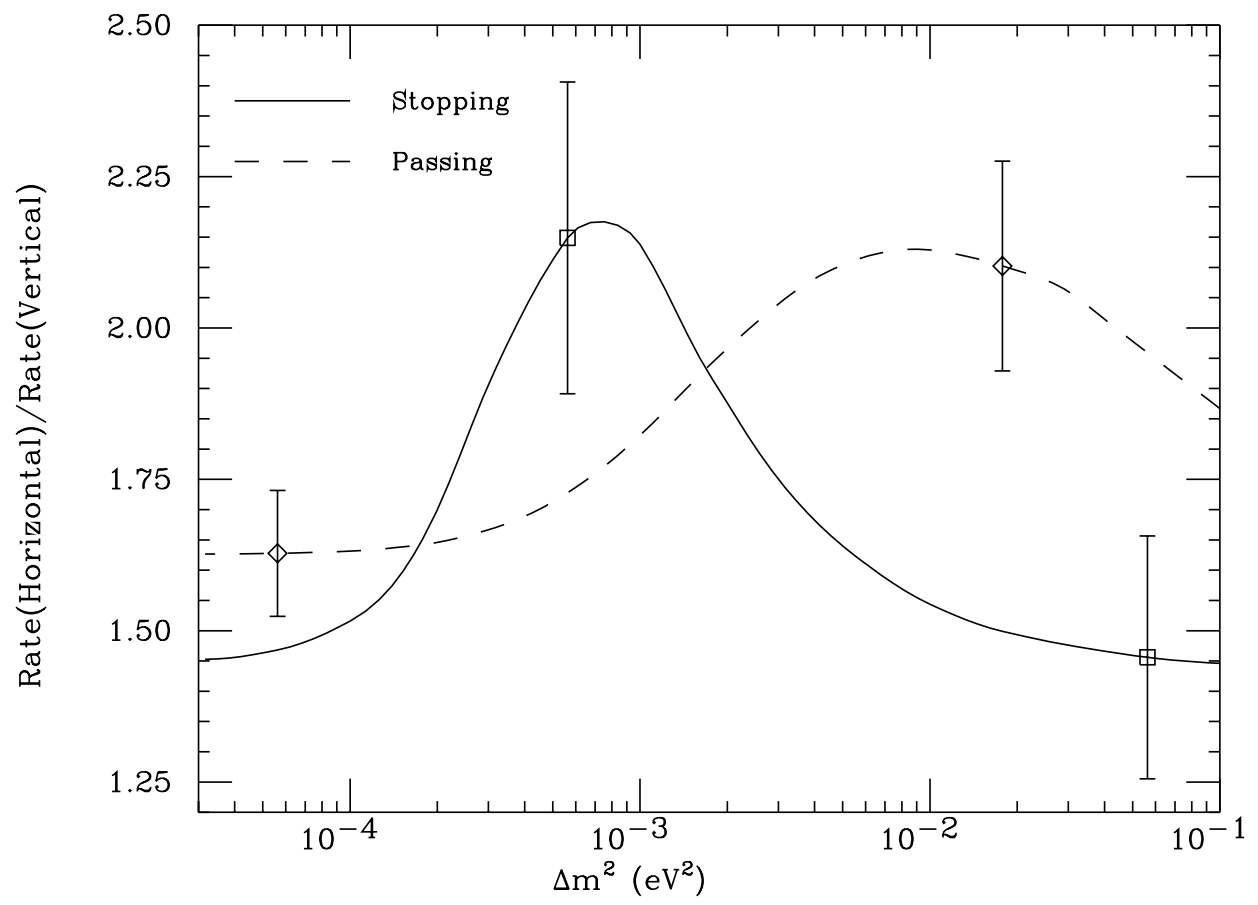

FIG. 2. Ratio of the 'horizontal' and 'vertical' muon rates for stopping and passing events in SuperKamiokande for $\nu_{\mu}-\nu_{\tau}$ oscillations with maximal mixing 


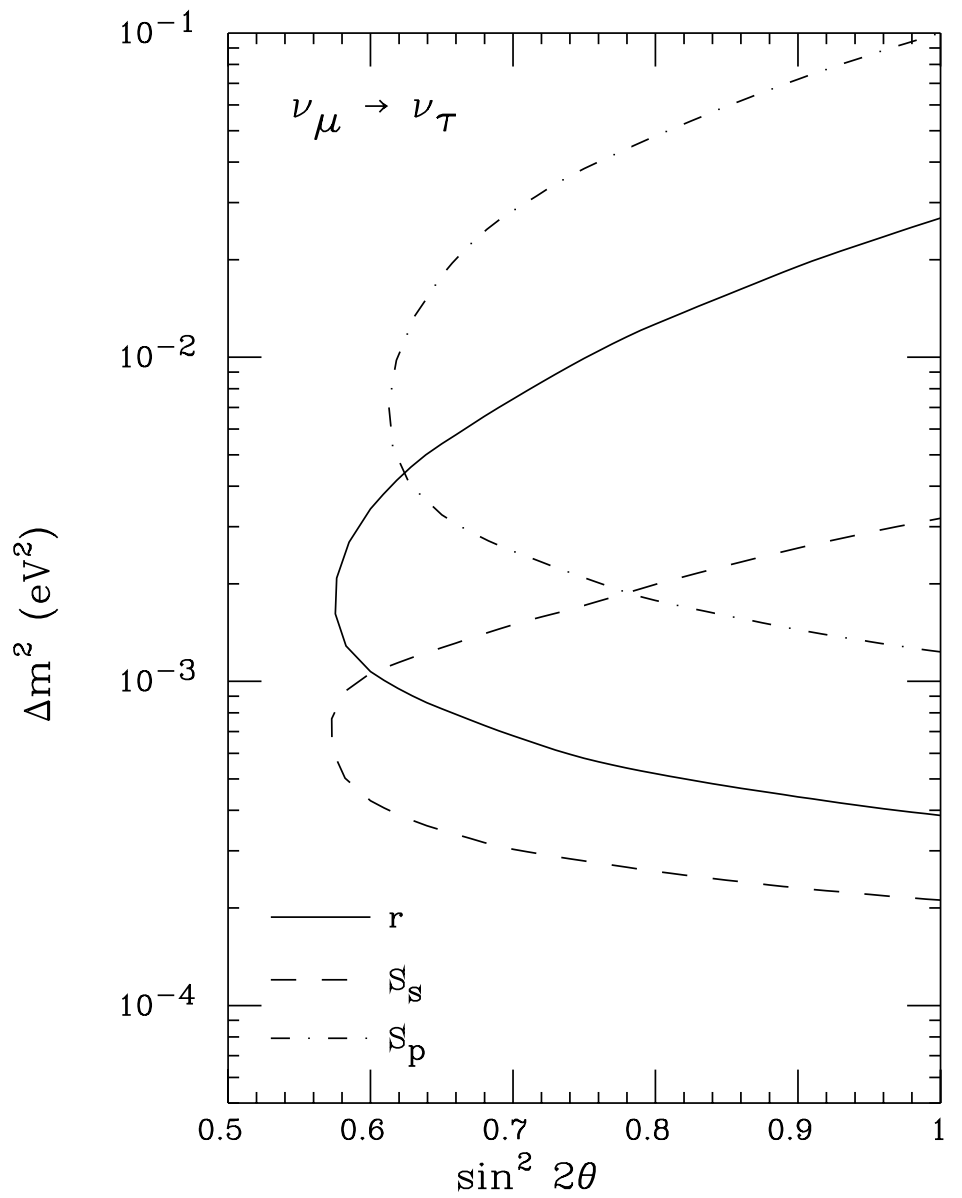

FIG. 3. Regions (to the right of the corresponding lines) in the $\left(\Delta m^{2}, \sin ^{2} 2 \theta_{\mu \tau}\right)$ plane, that can be excluded at $95 \%$ C.L. with two years of SuperKamiokande data from measurements of $r, S_{s}$ ans $S_{p}$. The exclusion curves are calculated assuming absence of oscillations and including theoretical errors of 8,3 and $4 \%$ for the predictions. 